\title{
Extract of Propolis on Resin-Modified Glass Ionomer Cement: Effect on Mechanical and Antimicrobial Properties and Dentin Bonding Strength
}

\author{
Narges Panahandeh (iD, ${ }^{1}$ Fatemeh Adinehlou, ${ }^{2}$ Seyedeh Mahsa Sheikh-Al-Eslamian (D), \\ and Hassan Torabzadeh ${ }^{3}$ \\ ${ }^{1}$ Dental Research Center, Research Institute for Dental Sciences, Shahid Beheshti University of Medical Sciences, \\ Tehran 1983963113, Iran \\ ${ }^{2}$ Private Practice, Tehran, Iran \\ ${ }^{3}$ Iranian Center for Endodontic Research, Research Institute of Dental Sciences, Shahid Beheshti University of Medical Sciences, \\ Tehran 1983963113, Iran \\ Correspondence should be addressed to Seyedeh Mahsa Sheikh-Al-Eslamian; mahsa.sheikh@gmail.com
}

Received 16 January 2021; Revised 17 March 2021; Accepted 29 March 2021; Published 12 April 2021

Academic Editor: Yingchao Su

Copyright $\odot 2021$ Narges Panahandeh et al. This is an open access article distributed under the Creative Commons Attribution License, which permits unrestricted use, distribution, and reproduction in any medium, provided the original work is properly cited.

\begin{abstract}
This study assessed the effect of addition of aqueous extract of propolis in different concentrations on the mechanical and antimicrobial properties of resin-modified glass ionomer cement (RMGIC). In this in vitro study, powder of Fuji II LC RMGIC was mixed with $25 \%$ and $50 \%$ aqueous extracts of propolis. Samples ( $n=15$ for shear bond strength, $n=5$ for flexural strength, and $n=20$ for the antibacterial activity test) were fabricated using this mixture. The buccal and lingual surfaces of 23 premolars were ground to expose dentin. Tygon tubes were filled with cement, bonded to dentin, and subjected to bond or the flexural strength test in a universal testing machine. Antibacterial activity was assessed using the disc diffusion and well-plate techniques against S. mutans. Data were analyzed using one-way ANOVA and Tukey's test. The three groups showed significant differences $(p<0.001)$. The 50\% propolis group had the lowest flexural and shear bond strength. The control group had the highest flexural and shear bond strength. No growth inhibition zone was noted around any of the discs. It can be concluded that addition of propolis to RMGIC did not confer any antibacterial activity against $S$. mutans and decreased the flexural and shear bond strength of RMGIC.
\end{abstract}

\section{Introduction}

Secondary caries refers to development of caries around or beneath the existing restorations over time [1] and is the most important and most common cause of restoration replacement [1-3]. Evidence shows that the type of restorative material significantly affects plaque accumulation and development of secondary caries [4], accumulation of Streptococcus mutans (S. mutans), and consequently, the risk of development of secondary caries is lower around glass ionomer (GI) restorations compared to composite resin [5]. However, no documented evidence exists regarding the inhibitory effects of GI cements on the occurrence of secondary caries [6]. In other words, GI cements have antibacterial effects on a narrow spectrum of microorganisms and have insignificant bactericidal activity [2]. Thus, researchers added chlorhexidine, antibiotics, and propolis to GI cements to enhance their antibacterial properties $[2,7-11]$.

GI cements have shortcomings such as moisture sensitivity and low primary strength [12]. However, chemical bonding to enamel and dentin, fluoride release potential, optimal biocompatibility, coefficient of thermal expansion similar to that of tooth structure, and absence of polymerization shrinkage are among the advantages of GI cements $[13,14]$. 
Addition of resin to GI cement increases its bond strength, physical properties, and moisture resistance and improves its esthetic appearance and polishability [15]. Resin-modified glass ionomer cements (RMGICs) are extensively used for patients at high risk of caries and those with multiple carious lesions due to their fluoride release potential and forming an actual chemical and micromechanical bond to tooth structure. They are also used as the restorative material of choice for restoration of cervical lesions [16]. A previous study on patients at high risk of caries due to xerostomia showed a significant reduction in the frequency of caries under RMGIC restorations compared to other restorations [17]. RMGICs have a bond strength similar or higher than that of conventional GI, which may be due to free acid-base reactions in RMGICs, that results in availability of polyacid for longer periods of time and formation of stronger bond.

Researchers are working on addition of chemical agents and plant extracts to dental materials to improve their mechanical and antibacterial properties. de Castilho et al. [18] in 2012 evaluated the effect of addition of chlorhexidine on biological and mechanical properties of RMGIC in vitro (antimicrobial, cytotoxic, and mechanical properties) and in vivo (microbiologic function in affected dentin when used for indirect pulp capping). The results showed that addition of $1.25 \%$ chlorhexidine to RMGIC significantly improved its antibacterial properties with no adverse effect on odontoblast-like cells or mechanical properties. Also, the use of mixture of RMGIC and chlorhexidine for indirect pulp capping resulted in complete elimination of S. mutans after 3 months.

Propolis is a natural resin produced by the honeybees [19] with antioxidative, antifungal, antiviral, and antibacterial properties [20]. It is used for treatment of candidiasis, acute necrotizing ulcerative gingivitis, gingivitis, periodontitis, and pulpitis in dentistry $[3,21]$. There are reports regarding the antibacterial effects of propolis on methicillinresistant Staphylococcus aureus [22, 23]. The bactericidal effects of propolis are attributed to the presence of cinnamic acid and flavonoids in its composition [24]. The ethanolic extract of propolis has been shown to have antifungal effects comparable to those of nystatin [25]. Antimicrobial effects of propolis on anaerobic oral bacteria [26], Staphylococcus aureus [27], Actinobacillus [28], and oral pathogenic microorganisms such as Streptococcus salivarius, Streptococcus sanguinis, Streptococcus mitis, and Candida albicans [29] have been previously documented. Also, a previous study showed that propolis had the greatest effect on Streptococcus mutans (S. mutans) among Gram-positive and Shigella among Gram-negative bacteria [30].

Propolis can have variable properties depending on the geographical region and vegetation of the area from which propolis has been collected as well as the season of collection. Its antimicrobial properties may also vary accordingly $[25,26,30]$. Up to now, about 300 chemical compositions have been identified in propolis, most notably flavonoids, phenols, and aromatic compounds. The ethanolic extract of propolis has been used in most previous studies [2, 7, 31-33].
The effect of addition of ethanolic extract of propolis on the mechanical and antimicrobial properties of conventional GI cement has been previously investigated [32]. GI is a waterbased cement, and it is clear that any additive should have been made on a water base composition; thus, this study aimed to assess the effect of addition of aqueous extract of Iranian propolis in different concentrations on the mechanical and antimicrobial properties of RMGIC.

\section{Materials and Methods}

2.1. Preparation of Aqueous Extract of Propolis. First, propolis was frozen and micronized using a ball mill. Micronized propolis was immediately added to boiling water in $1: 2$ ratio and stirred for one hour on indirect heat. After two days, the solution was filtered using a filter press. The obtained suspension was centrifuged at $8800 \mathrm{rpm}$ for 30 minutes to obtain the aqueous extract. The obtained extract had a dark brown color. The solution was stored in the dark until the experiment.

The powder to liquid ratio of cement was determined according to the manufacturer's instructions. Mixing was carried out on disposable pads using a spatula. Aqueous extract of propolis [34] in $25 \%$ and $50 \%$ concentrations $[7,35,36]$ was added to the RMGIC. The mixture was used for preparation of samples.

The shear bond strength test was performed on 23 extracted human premolars. The teeth were immersed in $0.5 \%$ chloramine $\mathrm{T}$ solution for disinfection for 24 hours and stored at $4^{\circ} \mathrm{C}$ until the experiment. Teeth were then sectioned mesiodistally with a microtome to divide into two halves and eliminate the enamel and expose the underneath dentin. Smooth surfaces were formed on the dentin surface when the specimens were lapped on 600- and 800-grit abrasive paper. Under a stereomicroscope, complete removal of the enamel and the smoothness of dentine surface were confirmed. The specimens were then rinsed off thoroughly with distilled water for at least $30 \mathrm{~s}$.

The study was approved in the Ethics Committee of School of Dentistry, Shahid Beheshti University of Medical Sciences (IR.SBMU.RIDS.Rec.1395.285).

2.2. Sample Preparation. Samples were fabricated of Fuji II LC RMGIC in pure form to serve as the control group and mixed with $25 \%$ and $50 \%$ aqueous extracts of propolis ( $n=45$ for the shear bond strength test, $n=15$ for the flexural strength test, and $n=60$ for the antibacterial activity test) (Dr. Jahangir Pharmaceutical and hygienic Co., Lorestan, Iran). For this purpose, RMGIC liquid and powder were mixed on disposable pads using a spatula. Aqueous extract of propolis in $25 \%$ and $50 \%$ concentrations was added to RMGIC where required.

2.3. Microshear Bond Strength Test. Rubber tubes (TBT, Tehran, Iran) with an internal diameter of $2.5 \mathrm{~mm}$ and $1 \mathrm{~mm}$ length were placed on dentin surfaces and filled with Fuji II LC RMGIC in pure form (control group) and the two 
mixtures of propolis. Light curing was performed for 20 seconds using a quartz tungsten halogen light curing unit (Turbo Co., Taipei, Taiwan) with a light intensity of $600 \mathrm{~mW} /$ $\mathrm{cm}^{2}$. The samples were then immersed in distilled water at $37^{\circ} \mathrm{C}$ for 24 hours. Before shear bond strength testing, cylindrical tubes were cut by a scalpel. The samples were then transferred to a universal testing machine (Zwick Roell, Ulm, Germany). The load at failure was recorded in Newton and converted to megapascal (MPa) by dividing the failure load by the cross-sectional area of each specimen.

2.4. Flexural Strength Test. Stainless steel molds measuring $25 \times 2 \times 2 \mathrm{~mm}$ were used for the fabrication of flexural samples. The molds were placed on glass plates and overfilled with RMGIC, and then, another glass plate was used with slight pressure on the mold in order to remove the excess material. Two Mylar strips were used to prevent adherence of samples to the plates. Samples were light cured in five overlapping areas from the core part to the sides. After removal from the mold, the samples were immersed in distilled water at $37^{\circ} \mathrm{C}$ for 24 hours. They were then transferred to a universal testing machine (Zwick Roell, Ulm, Germany). Load was applied to the sample at a crosshead speed of $1 \mathrm{~mm} /$ minute with $0.5 \mathrm{~N}$ preload. Maximum load at failure was recorded, and the flexural strength value was determined using the following formula: $\sigma=3 \mathrm{Fl} / 2 \mathrm{bh}^{2}$ ( $l$, distance between two jigs; $b$, specimen's width; $h$, specimen's height; and $\sigma$, flexural strength).

2.5. Assessment of Antibacterial Activity Using the Agar Diffusion Test. Plexiglass mold was used to fabricate twenty discs measuring $5 \mathrm{~mm}$ in diameter and $2 \mathrm{~mm}$ in thickness for each group (25\% and $50 \%$ aqueous extract of propolis and control). S. mutans (PTCC 1683) was used for the agar diffusion test. A $5 \mathrm{~mm}$ layer of agar was applied in Petri dishes. S. mutans suspension was prepared in $0.5 \mathrm{McF}$ arland standard concentration containing $1.5 \times 10^{8}$ bacteria, spread-cultured on blood agar, and incubated at $37^{\circ} \mathrm{C}$ for 24 hours. Discs were placed on the plates and incubated at $37^{\circ} \mathrm{C}$ for 48 hours. The diameter of growth inhibition zone around each disc was investigated.

To be assure of the antibacterial activity of propolis in pure form, the well-plate method was used. Blood agar culture medium and $S$. mutans were prepared as above. Using a pipette, two wells were created in agar; $30 \mu \mathrm{L}$ of propolis was added to one well and $30 \mu \mathrm{L}$ of ciprofloxacin was added to another well. The plates were then incubated at $37^{\circ} \mathrm{C}$ for 48 hours, and finally, antibacterial activity was recorded.

2.6. Statistical Analysis. Normal distribution of data was assessed using the Shapiro-Wilk test. The data were analyzed using one-way ANOVA. Tukey's test was applied for pairwise comparisons. All statistical analyses were performed using SPSS version 20 software (SPSS Inc., IL, USA) at $p<0.05$ level of significance.

\section{Results}

The Shapiro-Wilk test showed that data in all three groups were normally distributed $(p>0.05)$. Thus, the three groups were compared using the parametric one-way ANOVA test.

3.1. Shear Bond Strength Test Results. Table 1 provides the shear bond strength test results in the three groups. One-way ANOVA showed that the three groups were significantly different $(p<0.001)$. Thus, pairwise comparisons were performed using Tukey's HSD test, which showed that $25 \%$ and $50 \%$ propolis groups were not significantly different in terms of shear bond strength $(p=0.055)$. The two $25 \%$ propolis group and 50\% group had significantly lower shear bond strength than the control group $(p=0.035$ and $p=0.001$, respectively).

3.2. Flexural Strength Test Results. Table 1 provides the flexural strength test results in the three groups. One-way ANOVA showed that the three groups were significantly different $(p<0.001)$. Pairwise comparison of groups using Tukey's HSD test showed that the $50 \%$ propolis group had the lowest flexural strength, while the control group had the highest flexural strength; $50 \%$ propolis showed significantly lower flexural strength than $25 \%$ propolis $(p=0.006)$. The control group exhibited significantly higher flexural strength than $25 \%$ and $50 \%$ propolis.

3.3. Antibacterial Test Results. No growth inhibition zone was noted around the discs. However, pure propolis extract caused a growth inhibition zone with $11 \mathrm{~mm}$ diameter. The diameter of growth inhibition zone around ciprofloxacin was $16 \mathrm{~mm}$ (Figure 1).

Figure 2 shows the summery of the overall experimental design, methods used, and results obtained.

\section{Discussion}

At present, the use of extract of the material, instead of its isolated effective components, is preferred since it may have superior therapeutic efficacy due to the synergistic effect of its constituents [2]. The current study evaluated the effect of addition of aqueous extract of propolis in different concentrations on the mechanical and antimicrobial properties of RMGIC.

Knowledge about the mechanical properties of dental materials is important to predict their long-term behavior. In this study, flexural strength and shear bond strength tests were performed to assess the mechanical properties of RMGIC enriched with aqueous extract of propolis. Our results showed that the shear bond strength of $25 \%$ and $50 \%$ propolis groups was not significantly different; however, the $25 \%$ propolis group showed higher shear bond strength, and both values were significantly lower than that of shear bond strength of the control group $(p<0.05)$. The flexural strength test revealed that the $50 \%$ propolis group had the lowest and the control group had the highest flexural strength $(p<0.05)$. 
TABLE 1: Mean \pm SD shear bond strength and flexural strength values.

\begin{tabular}{|c|c|c|c|c|c|c|}
\hline \multirow{2}{*}{ Groups } & \multicolumn{3}{|c|}{ Shear bond strength $(\mathrm{MPa})$} & \multicolumn{3}{|c|}{ Flexural strength $(\mathrm{MPa})$} \\
\hline & ${ }^{*}$ Mean \pm SD & Minimum & Maximum & ${ }^{\dagger}$ Mean \pm SD & Minimum & Maximum \\
\hline Control (RMGI) & $2.37 \pm 1.29$ & 0.47 & 5.10 & $50.26 \pm 4.24$ & 45.10 & 54.16 \\
\hline $25 \%$ propolis & $1.43 \pm 1.08$ & 0.20 & 4.05 & $34.97 \pm 4.49$ & 30.10 & 41.40 \\
\hline $50 \%$ propolis & $0.55 \pm 0.46$ & 0.08 & 1.80 & $19.49 \pm 8.26$ & 10.91 & 28.50 \\
\hline
\end{tabular}

${ }^{*}$ Mean of 15 samples $(n=15) .{ }^{\dagger}$ Mean of 5 samples $(n=5)$.

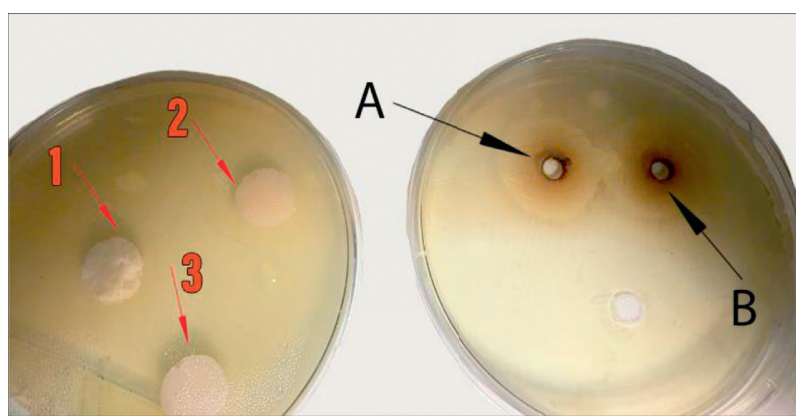

FiguRE 1: (a) Glass ionomer groups with (1) propolis $0 \%$, (2) propolis $25 \%$, and (3) propolis $50 \%$ showing no antibacterial activity against Streptococcus mutans. There was no significant difference between the groups $(p<0.05)$. (b) Antibacterial activity of ciprofloxacin $(\mathrm{A})$ and pure propolis (B) against Streptococcus mutans. The agar diffusion test showed an inhibition zone of $16 \mathrm{~mm}$ for ciprofloxacin and $11 \mathrm{~mm}$ for pure propolis.
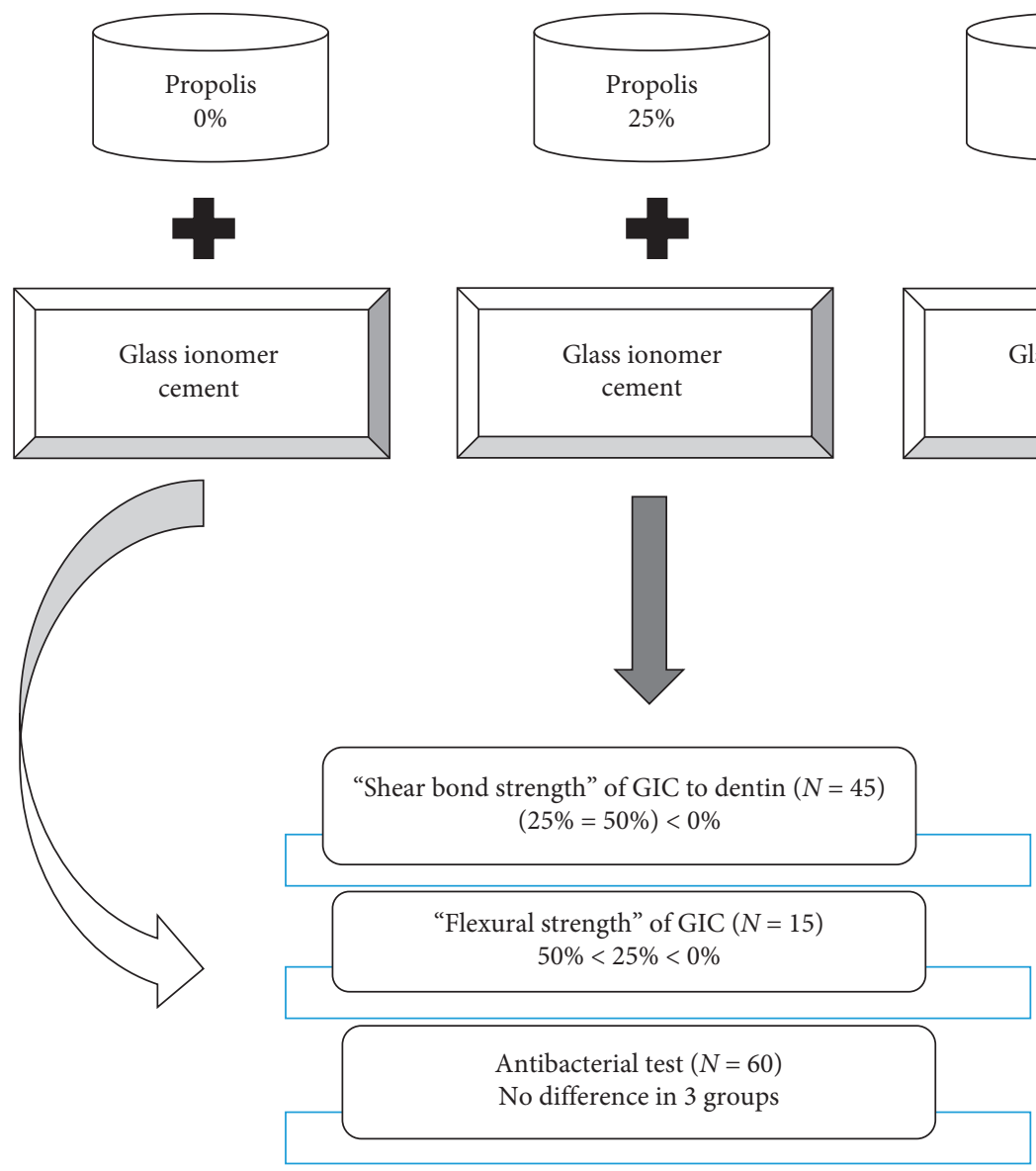

GIC: glass ionomer cement

Figure 2: Experimental design and the results of the study.
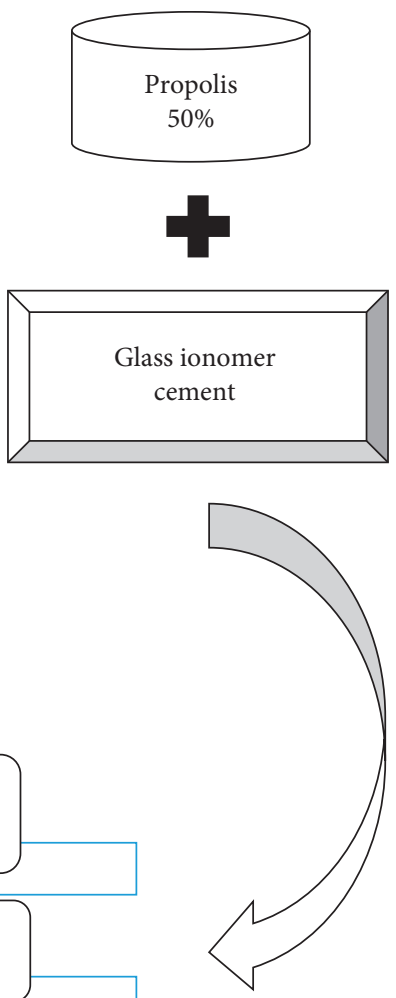
It is hypothesized that addition of propolis to RMGIC may increase its antibacterial properties without any deleterious effect on its mechanical features. The results showed that addition of aqueous extract of propolis negatively affected the mechanical properties and did not confer antibacterial activity to the cement (i.e., no growth inhibition zone was observed around the discs made of RMGIC enriched with propolis). However, pure propolis extract created a growth inhibition zone with $11 \mathrm{~mm}$ diameter.

Most previous studies on the effect of propolis on antimicrobial activity of GI cements have used ethanolic extract of propolis $[2,31,33]$. However, considering that the GI cements are water based, we assessed the effect of aqueous extract of propolis in this respect.

Some studies have shown that ethanolic extract of propolis increased the bond strength of GI [18, 36]. In contrast, Prabhakar et al. [32] compared the shear bond strength and fluoride release potential of conventional GI cement and GI cement mixed with $1 \%$ ethanolic extract of propolis and found no significant difference in shear bond strength of the two groups, while the fluoride release increased significantly. As ethanol may evaporate during setting, the powder: liquid ratio would not be affected by the incorporation of the ethanolic extract. On the other hand, the water in the aqueous extract of propolis will remain in the cement as loosely bond water which may result in decreased mechanical properties.

Evidence shows that propolis contains fatty acids and phenolic compounds [31,36]. Polyphenols have high molecular weight with unique properties [36]. When propolis is mixed with RMGIC, the hydroxyl phenolic group of propolis reacts with the carboxyl group of RMGIC $[32,36,37]$. In this process, propolis can serve as a space former for different carboxyl groups and create active crosslinks and sodium bridges. As the result of increased crosslinks, the complexity of GI cement increases. However, when aqueous extract of propolis is added to RMGIC, less amount of liquid reacts with powder and crosslinks increase. Since the GI liquid plays an important role in improving the working time and setting time of material, voids may form during mixing of cement with aqueous extract of propolis, which would negatively affect the mechanical properties $[2,36]$.

The result of this study showed growth inhibition zones with $11 \mathrm{~mm}$ and $16 \mathrm{~mm}$ diameters around pure propolis extract and ciprofloxacin, respectively. Although chlorhexidine is considered the material to be used as the positive control in the evaluation of antibacterial activity, there have been studies in which ciprofloxacin has been employed for the same purpose, e.g., an investigation by Saputo et al. used ciprofloxacin as a positive control group against Streptococcus mutans [38]. The effect of ethanolic extract of propolis on antibacterial properties of conventional GI cement was evaluated, and the results showed that addition of propolis enhanced antimicrobial properties of the cement $[2,7]$. Their results were different from the current study, which is probably due to the use of ethanol as a solvent instead of water. Type of solvent of propolis can significantly affect its antibacterial activity [31]. Udoye et al. [31] showed that ethanolic extract of propolis affects a wider spectrum of bacteria, and its bactericidal effect is greater than that of aqueous extract.

Some other factors may also affect the test results. For instance, application of liquid propolis in a well created in the plate caused a growth inhibition zone, while its incorporation into RMGIC and their placement on the agar plate as solid discs did not show any antimicrobial activity. This finding may indicate that release of antibacterial agents from liquids would occur faster and easier than from solid products. Accordingly, Yadiki et al. [37] demonstrated that due to the viscosity of Fuji IX cement and high density of the set cement, release of antibacterial agents from this material would be difficult.

\section{Conclusion}

Addition of propolis to RMGIC did not confer any antibacterial activity against $S$. mutans and in contrast decreased its flexural and shear bond strength.

\section{Data Availability}

The data used to support the findings of this study are available from the corresponding author upon request.

\section{Conflicts of Interest}

The authors declare that there are no conflicts of interest.

\section{Acknowledgments}

The authors are thankful to Dr Hussein Jahangir, the chief executor of Dr Jahangir Health Drug Company, and Dr Siamak Beiranvand, the R\&D manager of the company, because of their valuable help in providing propolis.

\section{References}

[1] I. A. Mjör, "Clinical diagnosis of recurrent caries," The Journal of the American Dental Association, vol. 136, no. 10, pp. 1426-1433, 2005.

[2] E. Hatunoğlu, F. Oztürk, T. Bilenler, S. Aksakallı, and N. Simşek, "Antibacterial and mechanical properties of propolis added to glass ionomer cement," The Angle Orthodontist, vol. 84, no. 2, pp. 368-373, 2014.

[3] A. Parolia, M. S. Thomas, M. Kundabala, and M. Mohan, "Propolis and its potential uses in oral health," International Journal of Medicine and Medical Science, vol. 2, no. 7, pp. 210-215, 2010.

[4] S. A. d. C. Duailibe, A. G. Gonçalves, and F. J. M. Ahid, "Effect of a propolis extract on Streptococcus mutans counts in vivo," Journal of Applied Oral Science, vol. 15, no. 5, pp. 420-423, 2007.

[5] A. Gama-Teixeira, M. R. L. Simionato, S. N. Elian, M. A. P. Sobral, and M. A. A. d. C. Luz, "Streptococcus mutans-induced secondary caries adjacent to glass ionomer cement, composite resin and amalgam restorations in vitro," Brazilian Oral Research, vol. 21, no. 4, pp. 368-374, 2007.

[6] T. Dérand, D. Birkhed, and S. Edwardsson, "Secondary caries related to various marginal gaps around amalgam restorations 
in vitro," Swedish Dental Journal, vol. 15, no. 3, pp. 133-138, 1991.

[7] N. Topcuoglu, F. Ozan, M. Ozyurt, and G. Kulekci, "In vitro antibacterial effects of glassionomer cement containing ethanolic extract of propolis on Streptococcus mutans," European Journal of Dentistry, vol. 6, no. 4, pp. 428-433, 2012.

[8] V. B. P. B. Troca, K. B. P. Fernandes, A. E. Terrile, M. C. Marcucci, F. B. d. Andrade, and L. Wang, "Effect of green propolis addition to physical mechanical properties of glass ionomer cements," Journal of Applied Oral Science, vol. 19, no. 2, pp. 100-105, 2011.

[9] A. R. Prabhakar, D. Prahlad, and S. R. Kumar, "Antibacterial activity, fluoride release, and physical properties of an antibioticmodified glass ionomer cement," Pediatric Dental, vol. 35, no. 5, pp. 411-415, 2013.

[10] S. Klai, M. Altenburger, B. Spitzmuller et al., "Antimicrobial effects of dental luting glass ionomer cements on Streptococcus mutans," ScientificWorld Journal, vol. 23, no. 807086, 2014.

[11] L. M. Marti, M. D. Mata, B. Ferraz-Santos, E. R. Azevedo, E. M. A. Giro, and A. C. C. Zuanon, "Addition of chlorhexidine gluconate to a glass ionomer cement: a study on mechanical, physical and antibacterial properties," Brazilian Dental Journal, vol. 25, no. 1, pp. 33-37, 2014.

[12] Y. Momoi, K. Hirosaki, A. Kohno, and J. F. McCabe, "Flexural properties of resin-modified "hybrid" glass-ionomers in comparison with conventional acid-base glass-ionomers," Dental Materials Journal, vol. 14, no. 2, pp. 109-119, 1995.

[13] S. Crisp and A. D. Wilson, "Reactions in glass ionomer cements," Journal of Dental Research, vol. 53, no. 6, pp. 1408-1413, 1974.

[14] S. Crisp and A. D. Wilson, "Reactions in glass ionomer cements: III. The precipitation reaction," Journal of Dental Research, vol. 53, no. 6, pp. 1420-1424, 1974.

[15] R. L. Sakaguchi, J. Ferracane, and J. M. Powers, Craig's Restorative Dental Materials-E-Book, Elsevier Health Sciences, Amsterdam, Netherlands, 2018.

[16] N. Frankel, G. J. Pearson, and R. Labella, "Long-term strength of aesthetic restoratives," Journal of Oral Rehabilitation, vol. 25 , no. 2, pp. 89-93, 1998.

[17] T. J. Hilton, J. L. Ferracane, and J. C. Broome, Summitt's Fundamentals of Operative Dentistry: A Contemporary Approach, Quintessence Publishing Company Incorporated, Batavia, NY, USA, 2013.

[18] A. R. F. de Castilho, C. Duque, T. d. C. Negrini et al., "In vitro and in vivo investigation of the biological and mechanical behaviour of resin-modified glass-ionomer cement containing chlorhexidine," Journal of Dentistry, vol. 41, no. 2, pp. 155-163, 2013.

[19] Z. Ahangari, M. Naseri, M. Jalili, Y Mansouri, F Mashhadiabbas, and A Torkaman, "Effect of propolis on dentin regeneration and the potential role of dental pulp stem cell in Guinea pigs," Cell Journal, vol. 13, no. 4, pp. 223-228, 2012.

[20] A. Kujumgiev, I. Tsvetkova, Y. Serkedjieva, V. Bankova, R. Christov, and S. Popov, "Antibacterial, antifungal and antiviral activity of propolis of different geographic origin," Journal of Ethnopharmacology, vol. 64, no. 3, pp. 235-240, 1999.

[21] R. O. Orsi, J. M. Sforcin, V. L. M. Rall et al., "Susceptibility profile of Salmonella against the antibacterial activity of propolis produced in two regions of Brazil," Journal of Venomous Animals and Toxins Including Tropical Diseases, vol. 11, no. 2, pp. 109-116, 2005.
[22] R. Raghukumar, L. Vali, D. Watson, J. Fearnley, and V. Seidel, "Antimethicillin-resistant Staphylococcus aureus (MRSA) activity of "pacific propolis" and isolated prenylflavanones," Phytotherapy Research, vol. 24, no. 8, pp. 1181-1187, 2010.

[23] Y. Onlen, N. Duran, E. Atik et al., "Antibacterial activity of propolis against MRSA and synergism with topical mupirocin," The Journal of Alternative and Complementary Medicine, vol. 13, no. 7, pp. 713-718, 2007.

[24] O. K. Mirzoeva, R. N. Grishanin, and P. C. Calder, "Antimicrobial action of propolis and some of its components: the effects on growth, membrane potential and motility of bacteria," Microbiological Research, vol. 152, no. 3, pp. 239-246, 1997.

[25] V. R. Santos, F. J. G. S. Pimenta, M. C. F. Aguiar, M. A. V. do Carmo, M. D. Naves, and R. A. Mesquita, "Oral candidiasis treatment with Brazilian ethanol propolis extract," Phytotherapy Research, vol. 19, no. 7, pp. 652-654, 2005.

[26] O. Koru, F. Toksoy, C. H. Acikel et al., "In vitro antimicrobial activity of propolis samples from different geographical origins against certain oral pathogens," Anaerobe, vol. 13, no. 34, pp. 140-145, 2007.

[27] S. Silici and S. Kutluca, "Chemical composition and antibacterial activity of propolis collected by three different races of honeybees in the same region," Journal of Ethnopharmacology, vol. 99, no. 1, pp. 69-73, 2005.

[28] F. Haghighati, S. Jafari, and E. J. M. Beyt, "Comparison of antimicrobial effects of ten Herbal extracts with chlorhexidine on three different oral pathogens; an in vitro study," Hakim Research Journal, vol. 6, 2003.

[29] M. L. Bruschi, E. H. G. Lara, C. H. G. Martins et al., "Preparation and antimicrobial activityof gelatin microparticles containing propolis against oral pathogens," Drug Development and Industrial Pharmacy, vol. 32, no. 2, pp. 229-238, 2006.

[30] A. Ugur and T. Arslan, "An in vitro study on antimicrobial activity of propolis from mugla province of Turkey," Journal of Medicinal Food, vol. 7, no. 1, pp. 90-94, 2004.

[31] C. I. Udoye, H. Jafarzadeh, and P. V. Abbott, "Transport media for avulsed teeth: a review," Australian Endodontic Journal, vol. 38, no. 3, pp. 129-136, 2012.

[32] A. R. Prabhakar, D. V. Balehosur, and N. Basappa, "Comparative evaluation of shear bond strength and fluoride release of conventional glass ionomer with $1 \%$ ethanolic extract of propolis incorporated glass ionomer cement-in vitro study," Journal of Clinical and Diagnostic Research, vol. 10, no. 5, p. 1, 2016.

[33] W. Krol, S. Scheller, J. Shani, G. Pietsz, and Z. Czuba, "Synergistic effect of ethanolic extract of propolis and antibiotics on the growth of staphylococcus aureus," ArzneimittelForschung, vol. 43, no. 5, pp. 607-609, 1993.

[34] L. Kubiliene, A. Jekabsone, M. Zilius et al., "Comparison of aqueous, polyethylene glycol-aqueous and ethanolic propolis extracts: antioxidant and mitochondria modulating properties," BMC Complementary and Alternative Medicine, vol. 18, no. 1, p. 165, 2018.

[35] E. Hatunoğlu, F. Öztürk, T. Bilenler, S. Aksakallı, and N. Şimşek, "Antibacterial and mechanical properties of propolis added to glass ionomer cement," The Angle Orthodontist, vol. 84, no. 2, pp. 368-373, 2013.

[36] M. Altunsoy, M. Tanrıver, U. Türkan, M. E. Uslu, and S. Silici, "In vitro evaluation of microleakage and microhardness of ethanolic extracts of propolis in different proportions added to glass ionomer cement," Journal of Clinical Pediatric Dentistry, vol. 40, no. 2, pp. 136-140, 2016. 
[37] J. V. Yadiki, S. R. Jampanapalli, S. Konda, H. C. Inguva, and V. K. Chimata, "Comparative evaluation of the antimicrobial properties of glass ionomer cements with and without chlorhexidine gluconate," International Journal of Clinical Pediatric Dentistry, vol. 9, no. 2, pp. 99-103, 2016.

[38] S. Saputo, R. C. Faustoferri, and R. G. Quivey, "A Drug repositioning approach reveals that $<$ span class="named-content genus-species" id=“named-content-1" $>$ Streptococcus mutans $<$ / span> is susceptible to a diverse range of established antimicrobials and nonantibiotics," Antimicrobial Agents and Chemotherapy, vol. 62, no. 1, pp. e01674-01617, 2018. 